\title{
Nutrition and Health in Mothers and Infants - Update from the Vitamin D Research Group
}

\author{
Karen O'Callaghan
}

School of Food and Nutritional Sciences, UCC

\section{Maternal Nutrition}

Nutrition plays an important role during all stages of the life cycle, from maternal health during pregnancy to healthy ageing in later life. Evidence suggests that a woman's diet during her pregnancy can not only have a substantial effect on the health of the growing baby, but is also related to a variety of health outcomes throughout childhood and even into adulthood. Put simply, by optimising the dietary intake of the expectant mother, we can help provide her child with a healthy start in life.

Since maternal nutrition at conception and throughout pregnancy directly influences the growth and development of her child, this provides a unique opportunity for health professionals to encourage women to make sensible dietary choices that will be of benefit to both themselves and their baby. Women are often motivated to improve their dietary and lifestyle behaviours at the beginning of pregnancy for the simple reason of wanting to deliver a healthy baby. It is therefore prudent that accurate, up-to-date information concerning nutrition and lifestyle behaviours is available to all pregnant women, in order to encourage women to make informed, conscious choices both peri and postnatally. Policy makers rely on the work of scientific researchers to provide the evidence that will guide recommendations for public health. High quality research studies are central for determining nutritional adequacy and are pivotal to the development of lifestyle intervention programmes that can positively impact the health of current and future generations.

Beginning at conception, the key "window of opportunity" continues at least throughout the first two years of a child's life. Pregnancy is a time of increased nutritional demand. The extra requirement for certain nutrients, specifically iron and folic acid, has been the focus of perinatal research for many years, and vitamin D has recently attracted much attention. However, despite the sudden growth in the scientific exploration of vitamin D and health, particularly regarding early life nutrition, dietary vitamin D requirements in pregnancy remain unknown. 


\section{The Vitamin D Focus}

The vitamin D status of the new-born infant is completely dependent on that of the expectant mother and so it is essential that women are nutritionally adequate during pregnancy. The term deficiency is based upon a biomarker of vitamin D status in blood serum. Vitamin D must reach a certain level in blood in order to prevent any symptoms of deficiency, but it is not yet known how much vitamin D pregnant women must consume to reach this specific threshold. My research aims to address this knowledge gap.

\section{Sources}

Commonly referred to as the "sunshine vitamin", vitamin D can be obtained from both dietary sources and from sunlight. Vitamin D is found naturally in few foods throughout the food supply, often in limited concentrations, and so dietary vitamin D intakes are typically low. Sunlight, therefore, is the most significant source of vitamin D for humans. Vitamin D production is stimulated through the action of ultraviolet B (UVB) radiation on the skin's surface, and subsequently stored within the body's adipose tissue (fat stores). The body can then draw upon this stored vitamin D when dietary supply and/or sunlight is limited. This becomes important during the winter months when the sunlight deficit results in inadequate vitamin D production. However, vitamin D synthesis is affected by several environmental factors, including latitude and cloud cover, which reduce the strength of the sun on the skin's surface and prevent vitamin D production. The combination of a poor dietary vitamin D intake and the typical 'Irish summer' mean that many of us are described as having a poor vitamin D status.

\section{Functions}

Vitamin D is both a nutrient and a hormone precursor. That is, it acts as a hormone within the body. Vitamin D is activated by specific enzymes in two consecutive reactions called 'hydroxylation', which refers to the addition of a water molecule during the activation process. In order to function as a hormone, vitamin D binds to what are known as 'vitamin D receptors' that are located in the body's tissues. Vitamin D receptors have long been found in skeletal tissue; however, the presence of such receptors in the placenta reveals a specific role for vitamin $D$ in pregnancy.

The most well-defined role for vitamin $\mathrm{D}$ is in the regulation of calcium metabolism, where vitamin D helps increase calcium absorption. When vitamin D intake is low, it can have a significant impact on bone health, leading to osteomalacia (softening of the bones) in adults and rickets in children. Rickets itself was once thought to be a disorder of the past, yet case reports from both Western and non-Western hospitals suggest that it is now making a comeback. Vitamin D-deficiency rickets is a disorder of the growth plate, resulting in bone anomalies and growth retardation in children. Children often present 
with muscle weakness and pain, or 'bowed legs' in severe cases. The bones are unable to fuse properly and so the knees turn outwards, giving a bow-like appearance to the legs. In the Western world, rickets is seen most often in children of a darker skin colour, where a low dietary vitamin $\mathrm{D}$ intake is coupled with inadequate production from UVB rays. Skin exposure to sunlight is limited by certain cultural and religious clothing practices, which can place some dark-skinned women and their children in the 'at-risk' group for low vitamin D status. Vitamin D-deficiency rickets is a nutritionally preventable disorder, and so its reoccurrence thus reflects the inadequacies in nutritional intake worldwide.

The prevention of rickets parallels the prevention of infant hypocalcaemia, where insufficient calcium status occurs due to inadequate intake or inhibition of absorption resulting from very low vitamin D status. Hypocalcaemia is a potentially life-threatening disorder, causing changes in heart beat and seizures in extreme cases. The dual role of vitamin D and calcium is therefore central to gestational health. There are, however, many other potential mechanisms by which vitamin D may influence maternal and fetal health, and results from vitamin $\mathrm{D}$ supplementation trials often support the relationship between maternal vitamin D intake and improved neonatal birth weight, length and growth during infancy. Emerging evidence also suggests a link between vitamin D deficiency at birth and childhood infectious diseases, cognitive disorders and long-term heart health, though the data is somewhat inconsistent.

\section{Requirements}

In Ireland, where the long winter and often cloud-covered summer is unable to compensate for an inadequate dietary vitamin D intake, supplemental vitamin D can contribute largely to overall vitamin D status. The question is, how much is enough?

"The most dangerous phrase in the English language is: We've always done it this way".

Rear Admiral Grace Hopper

In 2011, the Institute of Medicine issued a report which stated that the dietary vitamin D recommendations for pregnancy were to remain the same as for women who are not pregnant, on the basis that there was "insufficient evidence" to support otherwise. So although the beneficial effect of vitamin D during pregnancy has been extensively described, a lack of substantial scientific data exists on which to base dietary advice.

\section{Current Studies - My Research}

My research focuses on the importance of maternal nutrition during pregnancy with a strong emphasis on the role of vitamin D. I am currently working as part of the Vitamin D Research Group in UCC, where I am the co-investigator of a nutrition-based study that 
aims to guide vitamin D recommendations for pregnant women, both in Ireland and internationally. This study, known as the DMAT Study, forms part of a large collaborative project called ODIN, led by Prof Mairéad Kiely and Prof Kevin Cashman. The objective of ODIN is to establish food-based solutions for the eradication of vitamin D deficiency. DMAT is a double-blind, randomised controlled trial of vitamin D versus placebo. In simple terms, this means that participants in the study will be assigned (or randomised) to one of three doses of supplemental vitamin D and asked to take one supplement each day from the beginning to the end of their pregnancy. The supplements provided will contain either an active or inactive (placebo) form of the vitamin. The given doses of vitamin D are completely safe, similar to what would be found in a commercial vitamin D supplement or multivitamin. Hence, no risks are foreseen in this trial. Double-blind simply means that neither the participant nor the investigators will be aware of the dose to which the subject has been allocated. This is a preventative measure to ensure that any results or trial outcomes cannot be affected by bias from those involved in the study. This study will examine blood vitamin D levels of the participants throughout pregnancy, accounting for vitamin D intake from both habitual food choices and sun exposure. By comparing serum (blood) vitamin D levels of those consuming the highest amount of vitamin $\mathrm{D}$ to those in the lower categories, we will be able to estimate the correct dose of vitamin D needed by a woman each day throughout her pregnancy to ensure that both mother and infant are considered "vitamin D sufficient".

My work on this study will form the basis of my PhD where the primary objective will be to provide the evidence to guide dietary vitamin $\mathrm{D}$ recommendations during pregnancy. A secondary aim of my research is to use novel techniques to track measurements of arterial stiffness and blood pressure throughout pregnancy which I hope will add a piece to the complex puzzle that is pregnancy-related hypertension. Ultimately, my work will focus on modifiable nutritional and lifestyle factors that can lead to a healthier mother and baby.

DMAT is currently taking place in UCC, in conjunction with INFANT (Irish Centre for Fetal and Neonatal Translational Research) at Cork University Maternity Hospital, and will continue to recruit participants for a minimum of one year. By conducting this trial over a 12 month period, we can ensure that any variability in seasonal sun exposure (which influences vitamin D status) is accounted for. We hope to recruit approximately 200 women from the Cork area, who will be followed from early to late gestation. Participant inclusion is driven by the desire of women to have a comprehensive, informed understanding of the dietary behaviours that will influence the health of their children. INFANT, which is a world-leading perinatal research centre and the first of its kind in Ireland, allows for high quality maternal and child research studies to take place here in Cork, which can help improve pregnancy outcomes at the national and international level.

"No amount of funding or resources can make up for a lack of volunteers".

-NHS ‘Why We Do Research’ Campaign 
Maternal nutrition is at the heart of the health of future generations. Diet is a modifiable lifestyle factor; supplementation can work provided women remain compliant with supplement use. However, dietary recommendations need to be established based upon sound, scientific data from quality-led clinical trials. By focusing on research in early life, we can provide the evidence to help policy makers implement public health strategies that will positively affect the lives of women and children for years to come.

Karen $\mathrm{O}^{\prime}$ Callaghan is a PhD candidate in the School of Food and Nutritional Sciences under the supervision of Professor Mairéad Kiely. The author wishes to acknowledge funding from the European Commission 7th Framework Programme and to thank the DMAT Study team and all participants of the DMAT Study. 